\title{
Recursos humanos odontológicos y necesidades de tratamiento de caries en adolescentes de 12 años en Chile
}

\author{
Human resources in dentistry and treatment needs of caries in 12-year-old \\ teenagers in Chile
}

\author{
Cabello Ibacache $R^{1}$, Rodríguez Martínez $\mathrm{G}^{1}$, Tapia Crispi $R^{2}$, Jara Bahamondes $\mathrm{G}^{3}$, Soto Quina $\mathrm{L}^{4}$, Venegas Cid $\mathrm{C}^{5}$
}

\begin{abstract}
RESUMEN
El objetivo del estudio es estimar los recursos odontológicos para resolver las necesidades de tratamiento de los adolescentes de 12 años de Chile, pertenecientes a los niveles socio económicos alto, medio y bajo. El estudio se realizó a partir de los resultados del "Estudio Diagnóstico Nacional de Salud Bucal del Adolescente de 12 Años y Evaluación del Grado de Cumplimiento de los Objetivos Sanitarios de Salud Bucal 2000-2010", proyecto FONIS el que fue realizado en una muestra de 2.232 adolescentes de 12 años, con representación regional proporcional a la población nacional. Para estratificar por nivel socioeconómico este estudio se usó la clasificación y ranking de pobreza por comuna de MIDEPLAN y la caracterización por NSE de los establecimientos del Ministerio de Educación Se calculó el recurso humano necesario mediante la aplicación de estándares de rendimiento del ministerio de salud, comparando estos parámetros en el eje de nivel socioeconómico. Se observan diferencias estadísticamente significativas en las necesidades de tratamiento entre los NSE, concentrándose la mayor necesidad en los grupos de NSE bajo y medio.
\end{abstract}

Rev. Clin. Periodoncia Implantol. Rehabil. Oral Vol. 4(2); 45-49, 2011.

Palabras clave: Necesidades de tratamiento, caries, epidemiología.

\begin{abstract}
The aim of this study is to determine oral health care delivery team workforce necessary to tackle oral health treatment needs of 12-year-old adolescents from different socioeconomic status. The sample consisted of 2.232 individuals. There was a proportional representation of the Nation's regions. The Socioeconomic status (SES) was stratified through the MIDEPLAN's poverty municipality ranking and by means of description of SES of the selected school by the Ministry of Education. To determine oral health treatments needs we carried out dental examination and WHO diagnosis criteria were used. We calculated the oral health care delivery team workforce based on Ministry of Health recommendations and compare among SES. Differences upon health care delivery team workforce necessary to tackle oral health treatment needs of 12-year-old adolescents from different socioeconomic status were evident.
\end{abstract}

Rev. Clin. Periodoncia Implantol. Rehabil. Oral Vol. 4(2); 45-49, 2011.

Key words: Treatment needs, dental caries, epidemiology.

\section{INTRODUCCIÓN}

A nivel global una de las enfermedades de mayor prevalencia e incidencia en la población general, es la caries dental. Las naciones desarrolladas han experimentado en el último tiempo una tendencia continua de descenso de la caries dental, sin embargo altas prevalencias de caries se pueden observar en algunos grupos minoritarios de estas poblaciones, por ejemplo los migrantes ${ }^{(1,2,3)}$. Por otro lado se sugiere una tendencia inversa que se puede observar en los países en vía de desarrollo ${ }^{(4)}$, aunque algunos reportes no sustentan esta situación ${ }^{(5)}$. La caries dental se concentra en poblaciones pobres tanto en países en vías de desarrollo como en los industrializados( ${ }^{(6)}$. Estas características hacen que la caries dental se considere un problema significativo de salud pública(7).

Chile no es ajeno a esta realidad, lo que se demuestra en el estudio "Diagnóstico Nacional de Salud Bucal del Adolescente de 12 Años y Evaluación del Grado de Cumplimiento de los Objetivos Sanitarios de Salud Bucal 2000-2010", realizado el año 2007 por Soto y colaboradores ${ }^{(8)}$ con el respaldo del Fondo Nacional de Investigación en Salud (FONIS SA05I20085). En este estudio se observó que sólo el $37.5 \%$ de los adolescentes están libres de caries, con diferencias significativas entre niveles socio-económicos (NSE), sexo y localización urbano/rural (LUR). La historia de caries medida por el Índice COP-D, fue de 1.9, cuya distribución siguió el mismo patrón antes enunciado, encontrando que el índice COP-D en el NSE alto es de 0.66 , muy por debajo el promedio nacional, versus el 1.96 del NSE medio y el 2.22 del NSE bajo. En este estudio se concluye que los determinantes sociales son relevantes como indicadores de riesgo de sufrir caries dental(8). Existe amplia evidencia que sugiere que la condición social, es un fuerte predictor, tanto de morbilidad como de mortalidad en salud(9), asimismo, es aceptada la existencia de una asociación entre el estado de salud y el estatus social: individuos de mejor nivel socioeconómico disfrutan de una mejor salud ${ }^{(10,11,12)}$.

La cuantificación de las necesidades de tratamiento de una población es de gran valor en el nivel local, como también en el nacional e internacional dado que entrega información para el cálculo del recurso que se requiere para el desarrollo de un plan de salud bucodental en las condiciones actuales o previstas, siempre que se tengan en consideración la demanda de tales necesidades ${ }^{(13)}$. En este sentido la Organización Mundial de la Salud recomienda recoger información respecto de las necesidades de tratamiento por caries en las encuestas de vigilancia epidemiológica(13).

\footnotetext{
1. Magíster en Ciencias Odontológicas Mención Cariología. Departamento de Odontología Restauradora. Facultad de Odontología, Universidad de Chile. Chile.

2. Magíster en Salud Pública Mención Administración en Salud. Instituto Salud y Futuro, Universidad Andrés Bello. Chile.

3. Especialista en Salud Pública en Odontología. Facultad de Medicina. Escuela de Odontología, Universidad Diego Portales. Chile.

4. Licenciada en Salud Pública, Mención Epidemiología. Facultad de Medicina. Escuela de Odontología, Universidad Diego Portales. Chile.

5. Práctica Privada. Chile.
} 
Los objetivos sanitarios fijados en Chile para la década 20002010 perseguían tres objetivos principales en materia odontológica: el primero disminuir la caries dental en la población menor de 20 años logrando un índice COP-D de 1.9 en la población de 12 años de edad; el segundo objetivo planteado era aumentar la cobertura de atención odontológica de los menores de 20 años a un 50\%; y finalmente el tercer objetivo declaraba aumentar la cobertura de la fluoruración del agua potable a un $75 \%^{(14)}$. Particularmente el segundo objetivo perseguía acceder a resolver las necesidades de la población de menores de 20 años alcanzando un $50 \%$ de cobertura de atención odontológica. La evaluación del cumplimiento de estas metas indica que respecto al segundo objetivo que la cobertura de atención en los menores de 20 en el año 1999 era del $22 \%$ de los individuos beneficiarios del Fondo Nacional de Salud (FONASA) y en el año 2008 alcanzó el $22.5 \%$ de la población. Las principal estrategia desplegada en este sentido fue la priorización de la atención odontológica integral en las edades de mayor riesgo biológico, sobre la base de que los períodos de erupción de los dientes, tanto en la dentición temporal como definitiva, y a las edades en que las personas son más vulnerables a la educación en salud. En este sentido se priorizó la atención odontológica en las edades de 2, 4, 6 y 12 años de edad y las mujeres embarazadas ${ }^{(15)}$.

El permanente desafío que representa para Chile el acceso equitativo a la atención de salud, hace necesaria la distribución racional y focalizada de los recursos, lo que amerita un análisis de las necesidades de tratamiento en base a los estudios diagnósticos. De esta manera se puede lograr que la información generada a través de los estudios e investigaciones contribuya entre otros, a la estimación y orientación de la cantidad, calidad y distribución de los recursos humanos necesarios para resolverlas.

El objetivo de este estudio es estimar los recursos odontológicos requeridos para resolver, en el nivel primario de salud, las necesidades de tratamiento de caries dental en adolescentes de 12 años de distintos niveles socioeconómicos, de distinta condición urbana rural y sexo.

\section{MATERIAL Y MÉTODO}

Este estudio corresponde a un análisis secundario de los datos obtenidos en el "Estudio Diagnóstico Nacional de Salud Bucal del Adolescente de 12 años y Evaluación del Grado de Cumplimiento de los Objetivos Sanitarios de Salud Bucal 2000-2010"(8). Particularmente se analizan las necesidades de tratamiento derivadas de caries dental. EI diseño del estudio base corresponde a un estudio de prevalencia, cuya metodología se encuentra descrita por la Organización Mundial de la Salud (OMS) ${ }^{(13)}$. Brevemente respecto al estudio base, la muestra del estudio base fue construida mediante un método de muestreo aleatorio, con una técnica de muestreo estratificado por conglomerado. El universo del estudio se definió como toda la población chilena de 12 años, con exclusión de zonas de difícil acceso: Isla de Pascua, Juan Fernández y el Territorio Chileno Antártico. En la determinación del tamaño de muestra se consideró la prevalencia de caries dental de los últimos estudios que fue de un $63 \%$, con un nivel de de confianza del $95 \%$ y un nivel de error de estimación del $2 \%{ }^{(8)}$. La muestra examinada fue de 2.232 adolescentes de 12 años, con representación regional proporcional a la población nacional. En cada región se seleccionó al azar comunas urbanas y rurales. Para estratificar por nivel socioeconómico se usó la clasificación y ranking de pobreza por comuna de MIDEPLAN y la caracterización por NSE de los colegios del Ministerio de Educación en: Particulares Pagados, Particulares Subvencionados y Municipalizados, basados en el monto del arancel de matriculas y mensualidades de los establecimientos educacionales. Los sujetos seleccionados fueron sometidos a un examen bucal realizado por examinadores previamente entrenados y calibrados, con la colaboración de la Organización Panamericana de la Salud (OPS). Las necesidades de tratamiento fueron determinadas según los criterios de la $\mathrm{OMS}^{(13)}$, identificándose necesidades de sellantes de puntos y fisuras, obturaciones y exodoncias.

\section{Metodología para Estimación del Recurso Humano Necesario}

Los recursos humanos necesarios se estimaron para un periodo de 12 meses, en base a las necesidades de sellantes de puntos y fisuras, obturaciones de una o dos paredes y exodoncias establecidas por el estudio base, para lo cual se utilizó la media de las actividades, la que fue multiplicada por el total de la población de 12 años de acuerdo al Censo del año 2007, obteniéndose el número total de actividades necesarias a realizar. El valor encontrado fue dividido por el rendimiento, recomendado por las Orientaciones para la Programación en Red del año 2008 del Ministerio de Salud(16), obteniéndose de esta manera el total de horas odontólogo, las que dividieron por 1.840 horas, que corresponde al cálculo de 230 días hábiles anuales de jornadas de 8 horas laborales diarias. Se obtuvo así el número total de odontólogos anuales. Para el cálculo del numero de auxiliares paramédicos de odontología se multiplicó el número de odontólogos por 1.2 de acuerdo a las orientaciones ministeriales ${ }^{(17)}$.

\section{Limitaciones del Estudio}

Las necesidades de obturaciones complejas fueron excluidas del análisis ya que su resolución se realiza en el nivel secundario o de especialidades.

Los rendimientos corresponden a estándares ministeriales que eventualmente se ajustan localmente, según recursos humanos y equipamiento disponibles.

Los criterios diagnósticos en los que se fundan las necesidades de tratamiento y por consiguiente el recurso humano necesario para resolver corresponde a los criterios recomendados por la $\mathrm{OMS}^{(13)}$. Estos criterios consideran la presencia de una lesión de caries cuando se observa una cavitación. Por lo tanto se sub estima toda la necesidad de tratamiento de lesiones de caries en estadios más temprano. En el mismo sentido el uso de exámenes complementarios de radiología podría aumentar la necesidad de tratamiento. Esto último genera un impacto de sub estimación del recurso humano necesario para resolver la problemática de la caries dental.

\section{Análisis Estadísticos de los Datos}

Para las necesidades de tratamiento y el recurso humano se estimaron las medias y los intervalos de confianza del $95 \%$ correspondientes. Para la comparación de las necesidades de tratamiento y recurso humano necesario entre los NSE se utilizó análisis de varianza (ANOVA) y test de Bonferroni para comparaciones múltiples. Se presentan tablas de medias e intervalos de confianzas para las necesidades y recursos odontológicos entre condición urbana rural y sexo. Se consideró la distribución de la población por NSE, condición urbana rural y sexo que se utilizó en el estudio base ${ }^{(8)}$.

\section{RESULTADOS}

En la Tabla 1 se presentan las medias de dientes por adolescente de 12 años de edad con necesidades de tratamiento por caries con sus respectivos intervalos de confianza del $95 \%$. Los datos muestran que existen en promedio 3.57 (IC 95\% 3.45-3.69) dientes susceptibles de recibir sellantes, 0.75 (IC 95\% 0.69-0.79) piezas dentarias que requieren obturaciones y 0.06 (IC 95\% 0.05-0.07) exodoncias.

En la Tabla 2 se presenta la necesidad de recurso humano en la atención primaria de salud. La categoría que concentra la mayor demanda de odontólogos y auxiliares paramédicos de odontología corresponde a la necesidad de sellantes con aproximadamente 70 odontólogos año y 84 auxiliares año. Para abordar las necesidades de operatoria simple se requieren aproximadamente 39 odontólogos y 47 auxiliares odontológicos en jornadas de dedicación exclusiva durante un año. La necesidad de recurso humano para el desarrollo de actividades de exodoncia es de 1 odontólogo y 1 auxiliar al año.

Tabla 1. Promedio de necesidades de tratamiento odontológico por adolescente de 12 años

\begin{tabular}{|c|c|c|}
\hline Tratamiento & Media & Intervalos de Confianza \\
\hline Sellantes & 3.57 & $(3.45-3.69)$ \\
\hline $\begin{array}{l}\text { *Obturación } \\
\text { Operatoria } \\
\text { Simple (1 ó 2 paredes) }\end{array}$ & 0.75 & $(0.69-0.79)$ \\
\hline Exodoncias & 0.06 & $(0.05-0.07)$ \\
\hline
\end{tabular}

*Obturaciones de 1 y 2 paredes, de resolución en Atención Primaria de Salud. 
Tabla 2. Necesidades de Recursos Humanos Odontológicos para resolver tratamientos preventivos y recuperativos del adolescente de 12 años.

\begin{tabular}{|c|c|c|}
\hline \multicolumn{3}{|c|}{ Chile 2007 } \\
\hline Actividad & Odontólogo/Año & Auxiliar/Año \\
\hline Sellantes & 70 & 84 \\
\hline $\begin{array}{c}\text { Operatoria } \\
\text { Simple } \\
\text { (1 ó 2 paredes) }\end{array}$ & 39 & 47 \\
\hline Exodoncias & 1 & 1 \\
\hline Total & 110 & 132 \\
\hline
\end{tabular}

En la Tabla 3, se observa que, para las necesidades de aplicación de sellantes, existen diferencias significativas entre el NSE alto y los NSE medio y bajo. No existen diferencias significativas entre estos dos últimos. De igual manera para la necesidades de tratamiento de operatoria simple, existen diferencias significativas $(p<0.01)$ entre el NSE alto y los NSE medio y bajo. No se observan diferencias significativas entre estos dos últimos NSE. Para la necesidad de tratamiento de exodoncia las diferencias se observan entre el NSE bajo y los niveles NSE medio y alto, sin observar diferencias entre estos últimos.

Tabla 3. Necesidades de tratamiento por Nivel Socioeconómico (NSE) del adolescente de 12 años.

\begin{tabular}{|c|c|c|c|c|c|}
\hline \multicolumn{5}{|c|}{$\begin{array}{c}\text { Intervalo de } \\
\text { confianza al } 95 \%\end{array}$} & \multirow[t]{2}{*}{$\mathrm{n}$} \\
\hline $\begin{array}{c}\text { Nivel } \\
\text { Socioeconómico }\end{array}$ & & Media & Inferior & Superior & \\
\hline \multirow{3}{*}{ NSE Alto } & $\begin{array}{c}\text { Tratamiento } \\
\text { Sellantes } \\
\end{array}$ & $2.9^{\mathrm{a}, \mathrm{b}}$ & 2.61 & 3.19 & \multirow{3}{*}{334} \\
\hline & $\begin{array}{l}\text { Tratamiento } \\
\text { de Exodoncia }\end{array}$ & $0.006^{\mathrm{c}}$ & -0.002 & .01 & \\
\hline & $\begin{array}{c}\text { Tratamiento } \\
\text { Operatoria simple }\end{array}$ & $0.24^{e f}$ & 0.17 & 0.32 & \\
\hline \multirow{3}{*}{ NSE Medio } & $\begin{array}{l}\text { Tratamiento } \\
\text { Sellantes } \\
\end{array}$ & $3.85^{\mathrm{a}}$ & 3.63 & 4.06 & \multirow{3}{*}{732} \\
\hline & $\begin{array}{c}\text { Tratamiento } \\
\text { de Exodoncia }\end{array}$ & $0.04^{d}$ & 0.02 & 0.06 & \\
\hline & $\begin{array}{c}\text { Tratamiento } \\
\text { Operatoria simple } \\
\end{array}$ & $0.78^{\mathrm{e}}$ & 0.68 & 0.87 & \\
\hline \multirow{3}{*}{ NSE Bajo } & $\begin{array}{l}\text { Tratamiento } \\
\text { Sellantes }\end{array}$ & $3.59^{b}$ & 3.43 & 3.75 & \multirow{3}{*}{1166} \\
\hline & $\begin{array}{c}\text { Tratamiento } \\
\text { de Exodoncia }\end{array}$ & $0.08^{c, d}$ & 0.06 & 0.1 & \\
\hline & $\begin{array}{c}\text { Tratamiento } \\
\text { Operatoria simple }\end{array}$ & $0.87^{f}$ & 0.79 & 0.95 & \\
\hline
\end{tabular}

$\mathrm{a}, \mathrm{b}, \mathrm{c}, \mathrm{d}, \mathrm{e}, \mathrm{f}=\mathrm{p}<0.01$. Diferencias observadas estadísticamente significativas mediante ANOVA y test de comparaciones múltiples de Bonferronni.

En la Tabla 4 se presenta la necesidad de recurso humano odontólogo en la atención primaria de salud, por nivel socioeconómico. EI NSE que concentra la mayor demanda de odontólogos corresponde al NSE bajo con aproximadamente 61 odontólogos año, en el cual más de la mitad de esta necesidad de recurso humano estaría destinado a la aplicación de sellantes, mientras que el NSE medio necesitaría aproximadamente 38 odontólogos año, y con la menor demanda de odontólogo año se presenta el NSE alto, con 10.35 odontólogo año.

Tabla 4. Recursos Humanos necesarios para resolver las necesidades de tratamiento por Nivel Socioeconómico (NSE) del adolescente de 12 años.

\begin{tabular}{|l|c|c|c|}
\hline & \multicolumn{3}{|c|}{ Odontólogos Año } \\
\hline Tratamiento & NSE Alto & NSE Medio & NSE Bajo \\
\hline Sellantes & 8.46 & 24.56 & 36.45 \\
\hline $\begin{array}{l}\text { Operatoria } \\
\text { simple }\end{array}$ & 1.87 & 13.27 & 23.55 \\
\hline Exodoncias & 0.02 & 0.34 & 1.08 \\
\hline
\end{tabular}

Los resultados muestran diferencias estadísticamente significativas en las necesidades de tratamiento odontológico, y por tanto del recurso humano necesario para resolverlas, entre los niveles socioeconómicos, principalmente entre el alto y el medio, y entre el alto y el bajo, lo que da cuenta de las inequidades en este campo, producto del menor daño que presenta el NSE alto, consecuente con el mejor manejo de los factores de riesgo y del mejor acceso a la atención odontológica, que tiene este estrato social.

Respecto de las necesidades de tratamiento y la condición urbano rural y de sexo (Tabla 5) los datos sugieren que existen diferencias significativas entre individuos rurales y urbanos. Los individuos rurales presentan menores necesidades de sellantes mientras que requieren de mayor tratamiento de operatoria simple. En relación al sexo existe una tendencia estadística de diferencia en relación a la necesidad de tratamiento de operatoria simple que indica que las mujeres poseen 0.8 dientes en promedio con necesidad terapéutica, mientras que los hombres presentan 0.69 dientes en esta condición.

Tabla 5. Necesidades de tratamiento por sexo y por condición urbano rural del adolescente de 12 años.

\begin{tabular}{|l|c|c|c|c|}
\hline \multirow{2}{*}{} & \multicolumn{2}{|c|}{ Condición Urbano Rural } & \multicolumn{2}{c|}{ Sexo } \\
\cline { 2 - 5 } & $\begin{array}{c}\text { Urbano } \\
(\mathbf{n}: 1896)\end{array}$ & $\begin{array}{c}\text { Rural } \\
(\mathbf{n}: 336)\end{array}$ & $\begin{array}{c}\text { Hombre } \\
(\mathbf{n}: 1093)\end{array}$ & $\begin{array}{c}\text { Mujer } \\
(\mathbf{n}: 1139)\end{array}$ \\
\cline { 2 - 5 } & \multicolumn{4}{|c|}{ Media (IC95\%) } \\
\hline $\begin{array}{l}\text { Tratamiento } \\
\text { Sellantes }\end{array}$ & $3.69(3.55-3.82)$ & $2.94(2.69-3.2)$ & $3.38(3.22-3.54)$ & $3.7(3.6-3.94)$ \\
\hline $\begin{array}{l}\text { Tratamiento } \\
\text { de Exodoncia }\end{array}$ & $0.05(0.04-0.07)$ & $0.09(0.05-0.13)$ & $0.06(0.05-0.08)$ & $0.05(0.04-0.07)$ \\
\hline $\begin{array}{l}\text { Tratamiento } \\
\text { Operatoria simple }\end{array}$ & $0.69(0.64-0.75)$ & $1.05(0.9-1.22)$ & $0.69(0.62-0.77)$ & $0.8(0.72-0.88)$ \\
\hline
\end{tabular}

Los recursos humanos necesarios para resolver las necesidades se concentran en la población urbana dado el número mayor de individuos en esta condición y la principal necesidad que concentra estos recursos corresponde a la necesidades de tratamientos de sellantes (Tabla 6).

Tabla 6. Recursos Humanos necesarios para resolver las necesidades de tratamiento por sexo y condición urbano rural del adolescente de 12 años.

\begin{tabular}{|l|c|c|c|c|}
\hline \multirow{2}{*}{} & \multicolumn{2}{|c|}{ Condición Urbano Rural } & \multicolumn{2}{c|}{ Sexo } \\
\cline { 2 - 5 } & $\begin{array}{c}\text { Urbano } \\
(\mathbf{n}: 1896)\end{array}$ & $\begin{array}{c}\text { Rural } \\
(\mathbf{n}: \mathbf{3 3 6})\end{array}$ & $\begin{array}{c}\text { Hombre } \\
(\mathbf{n}: 1093)\end{array}$ & $\begin{array}{c}\text { Mujer } \\
(\mathbf{n}: 1139)\end{array}$ \\
\cline { 2 - 5 } & \multicolumn{2}{|c|}{ MEDIA (IC95\%) DE INDIVIDUOS NECESARIOS } \\
\hline $\begin{array}{l}\text { Tratamiento } \\
\text { Sellantes }\end{array}$ & 60.93 & 8.63 & 33.45 & 35.35 \\
\hline $\begin{array}{l}\text { Tratamiento } \\
\text { de Exodoncia }\end{array}$ & $(58.62-63.08)$ & $(7.9-9.4)$ & $(31.86-35.03)$ & $(34.39-37.64)$ \\
\hline $\begin{array}{l}\text { Tratamiento } \\
\text { Operatoria simple }\end{array}$ & $\begin{array}{c}1.10 \\
(28.18-38-1.54)\end{array}$ & $\begin{array}{c}0.35 \\
(0.2-0.51)\end{array}$ & $\begin{array}{c}0.79 \\
(0.66-1.06)\end{array}$ & $\begin{array}{c}0.64 \\
(0.51-0.89)\end{array}$ \\
\hline
\end{tabular}

Las tendencias de mayor concentración de necesidades en la mujer y en la ruralidad se observan en las Tablas 7 y 8 . Así mismo los recursos se concentran en mujeres urbanas donde se conjugan en la mayor necesidad y la concentración de mayor número de población. 
Tabla 7. Necesidades de tratamiento por sexo y condición urbano rural del adolescente de 12 años.

\begin{tabular}{|c|c|c|c|c|c|c|}
\hline \multirow[b]{2}{*}{ Sexo } & \multirow[t]{2}{*}{$\begin{array}{c}\text { Condición } \\
\text { urbano rural }\end{array}$} & & \multicolumn{3}{|c|}{$\begin{array}{c}\text { Intervalo de } \\
\text { confianza del } 95 \%\end{array}$} & \multirow[t]{2}{*}{$\mathbf{n}$} \\
\hline & & & Media & Inferior & Superior & \\
\hline \multirow[t]{6}{*}{ Hombre } & \multirow[t]{3}{*}{ Urbano } & \begin{tabular}{|l|} 
Tratamiento \\
Sellantes
\end{tabular} & 3.49 & 3.31 & 3.67 & \multirow{3}{*}{917} \\
\hline & & $\begin{array}{l}\text { Tratamiento } \\
\text { de Exodoncia }\end{array}$ & 0.05 & 0.04 & 0.07 & \\
\hline & & \begin{tabular}{|l|} 
Tratamiento \\
Operatoria simple
\end{tabular} & 0.62 & 0.54 & 0.69 & \\
\hline & \multirow[t]{3}{*}{ Rural } & \begin{tabular}{|l|} 
Tratamiento \\
Sellantes
\end{tabular} & 2.8 & 2.47 & 3.13 & \multirow{3}{*}{176} \\
\hline & & $\begin{array}{l}\text { Tratamiento } \\
\text { de Exodoncia }\end{array}$ & 0.1 & 0.05 & 0.16 & \\
\hline & & \begin{tabular}{|l|} 
Tratamiento \\
Operatoria simple
\end{tabular} & 1.09 & 0.86 & 1.32 & \\
\hline \multirow[t]{6}{*}{ Mujer } & \multirow[t]{3}{*}{ Urbano } & \begin{tabular}{|l|} 
Tratamiento \\
Sellantes
\end{tabular} & 3.87 & 3.67 & 4.06 & \multirow{3}{*}{97} \\
\hline & & $\begin{array}{l}\text { Tratamiento } \\
\text { de Exodoncia }\end{array}$ & 0.05 & 0.03 & 0.07 & \\
\hline & & \begin{tabular}{|l|} 
Tratamiento \\
Operatoria simple
\end{tabular} & 0.76 & 0.68 & 0.84 & \\
\hline & \multirow[t]{3}{*}{ Rural } & \begin{tabular}{|l|} 
Tratamiento \\
Sellantes \\
\end{tabular} & 3.1 & 2.71 & 3.49 & \multirow{3}{*}{160} \\
\hline & & $\begin{array}{l}\text { Tratamiento } \\
\text { de Exodoncia }\end{array}$ & 0.08 & 0.03 & 0.133 & \\
\hline & & \begin{tabular}{|l|} 
Tratamiento \\
Operatoria simple
\end{tabular} & 1.01 & 0.78 & 1.25 & \\
\hline
\end{tabular}

Tabla 8. Recursos Humanos necesarios para resolver las necesidades de tratamiento por sexo y condición urbano rural del adolescente de 12 años.

\begin{tabular}{|c|c|c|c|c|c|c|}
\hline \multirow[b]{2}{*}{ Sexo } & \multirow[t]{2}{*}{$\begin{array}{c}\text { Condición } \\
\text { urbano rural }\end{array}$} & & \multicolumn{3}{|c|}{$\begin{array}{c}\text { Intervalo de } \\
\text { confianza del } 95 \%\end{array}$} & \multirow[t]{2}{*}{$\mathbf{n}$} \\
\hline & & & Media & Inferior & Superior & \\
\hline \multirow[t]{6}{*}{ Hombre } & \multirow[t]{3}{*}{ Urbano } & $\begin{array}{l}\text { Tratamiento } \\
\text { Sellantes }\end{array}$ & 27.89 & 26.45 & 29.33 & \multirow{3}{*}{917} \\
\hline & & $\begin{array}{l}\text { Tratamiento } \\
\text { de Exodoncia }\end{array}$ & 0.53 & 0.43 & 0.75 & \\
\hline & & $\begin{array}{l}\text { Tratamiento } \\
\text { Operatoria simple }\end{array}$ & 13.21 & 11.51 & 14.70 & \\
\hline & \multirow[t]{3}{*}{ Rural } & $\begin{array}{l}\text { Tratamiento } \\
\text { Sellantes }\end{array}$ & 4.29 & 3.79 & 4.80 & \multirow{3}{*}{176} \\
\hline & & $\begin{array}{l}\text { Tratamiento } \\
\text { de Exodoncia }\end{array}$ & 0.20 & 0.10 & 0.33 & \\
\hline & & $\begin{array}{l}\text { Tratamiento } \\
\text { Operatoria simple }\end{array}$ & 4.46 & 3.52 & 5.40 & \\
\hline \multirow[t]{6}{*}{ Mujer } & \multirow[t]{3}{*}{ Urbano } & $\begin{array}{l}\text { Tratamiento } \\
\text { Sellantes }\end{array}$ & 33.01 & 31.31 & 34.64 & \multirow{3}{*}{979} \\
\hline & & $\begin{array}{l}\text { Tratamiento } \\
\text { de Exodoncia }\end{array}$ & 0.57 & 0.34 & 0.80 & \\
\hline & & $\begin{array}{l}\text { Tratamiento } \\
\text { Operatoria simple }\end{array}$ & 17.29 & 15.47 & 19.11 & \\
\hline & \multirow[t]{3}{*}{ Rural } & $\begin{array}{l}\text { Tratamiento } \\
\text { Sellantes }\end{array}$ & 4.32 & 3.78 & 4.87 & \multirow{3}{*}{160} \\
\hline & & $\begin{array}{l}\text { Tratamiento } \\
\text { de Exodoncia }\end{array}$ & 0.15 & 0.06 & 0.25 & \\
\hline & & $\begin{array}{l}\text { Tratamiento } \\
\text { Operatoria simple }\end{array}$ & 3.75 & 2.90 & 4.65 & \\
\hline
\end{tabular}


que abre una oportunidad innegable de complementar las acciones ejecutadas por odontólogos, para que sean realizadas bajo supervisión, por el personal auxiliar, sobre todo considerando que aproximadamente el $62 \%$ de las horas odontólogo necesarias para cerrar la brecha de atención de los adolescentes de niveles medio y bajo, se concentran en acciones de prevención, que pueden ser realizadas por las auxiliares dentales.

Por último, atendiendo a la vulnerabilidad de las caries dentales a las medidas de promoción y prevención y a la proyección que el daño de salud bucal tiene en el tiempo revela lo importante que es invertir en las población durante la niñez y adolescencia, para permitir a futuro cohortes de adultos que gocen de una buena calidad de vida, con el consiguiente aumento de su autoestima y herramientas saludables que contribuyan a su superación y a mejorar sus fuentes de trabajo y bienestar.

\section{CONFLICTOS DE INTERÉS}

El autor declara que él y los coautores no sufren conflicto de interés con los resultados publicados.

\section{REFERENCIAS BIBLIOGRÁFICAS}

1. Irigoyen ME, Maupomé G, Mejia AM. Caries experience and treatment needs in a 6-to-12-year-old urban child population in relation to socioeconomic status. Community Dent Health, 1999; 16: 245-249.

2. Irigoyen ME, Luengas IF, Yashine A, Mejia AM, Maupomé G. Dental caries experience in Mexican schoolchildren from rural and urban communities. Int Dental J, 2000; 50: 41-45.

3. Marthaler TM. Changes in dental caries 1953-2003. Caries Res, 2004; 38: 173-181.

4. Winter GB. Epidemiology of dental caries. Arch Oral Biol, 1990; 35 (Suppl.): 1S-7S.

5. Cleaton-Jones $\mathrm{P}$, Fatti $\mathrm{P}$, Bonecker $\mathrm{M}$. Dental caries trends in 5-to 6-year-old and 11-to-13-year-old children in three UNICEF designated regions-Sub Saharan Africa, Middle East and North Africa, Latin America and Caribbean: 1970-2004. Int Dent J, 2006; 56: 294-300.

6. Donahue GJ, Waddell N, Plough AL, del Águila MA, Garland TE. The ABCDs of treating the most prevalent childhood disease. Am J Public Health, 2005; 95: 1322-1324.

7. Petersen P. The World Oral Health report 2003: continuous improvement of oral health in the $21^{\text {st }}$ century - the approach of the WHO Global Oral Health Programme. Community Dent Oral Epidemiol, 2003; 31 (Suppl 1): 3-24.

8. Soto L, Tapia R, Jara G, Rodríguez G, Urbina T. Diagnóstico nacional de salud bucal del adolescente de 12 años y evaluación del grado de cumplimiento de los Objetivos Sanitarios de Salud Bucal 2000-2010. Ediciones Universidad Mayor. 2007.

9. Subramanian $\mathrm{V}$ et al. Inequidad de ingreso y autopercepción de salud: Un análisis desde la perspectiva contextual en las comunas chilenas. Rev Méd Chile [online], 2003; 131(3): 321-330.ISSN0034-9887. doi:10.4067/ S0034-98872003000300012.

10. Kawachi I. Income inequality in health. Chap. 4. In: Berkman L, Kawachi I (Eds.). Social Epidemiology. New York: Oxford University Press; 2000, p. 76-93.
11. Lynch J, Kaplan G. Socioeconomic position, Chap. 2. In: Berkman L, Kawachi I (Eds.). Social Epidemiology. New York: Oxford University Press; 2000, p. 13-35.

12. Marmot M, Wilkinson RG. Psychosocial and material pathways in the relation between income and health: A response to Lynch, et al. BMJ, 2001; 322: 1233-1236.

13. Encuestas de Salud Bucodental. Métodos Básicos. Cuarta edición. Organización Mundial de la Salud/Organización Panamericana de la Salud. Cuarta edición Ginebra 1997. ISBN 924354493.

14. Objetivos Sanitarios para la Década 2000-2010. Evaluación a mitad del período. Estado de Avance en los objetivos de impacto. Subsecretaría de Salud Pública. Ministerio de Salud, Chile. 2006.

15. Los Objetivos Sanitarios para la Década 2000-2010 Evaluación de final del período,Grado de cumplimiento de los objetivos de Impacto. Ministerio de Salud. Gobierno de Chile. 2011.

16. Petersen PE, Ogawa $\mathrm{H}$. Strenghtening the prevention of periodontal disease: The WHO approach. J Periodontol, 2005; 76: 2187-2193.

17. Orientaciones para la Programación en Red Año 2008-Subsecretaría de Redes Asistenciales/División de Gestión de la Red Asistencial/División de Atención Primaria, Ministerio de Salud, Chile.

18. Informe Catastro Nacional de Recursos Humanos Odontológicos SNSS-2003, Departamento de salud Bucal, Ministerio de Salud, Chile.

19. Baelum V et al. Chapter 32: "For richer,for poorer, in sickness and in health...". The role of dentistry in controlling caries and periodontitis globally. Dental Caries, 2008; 2: 575-605, Blackwell Munksgaard.

20. Orientaciones para la Planificación y Programación en Red Año 2010 Subsecretaría de Redes Asistenciales, Ministerio de Salud, Chile. 